Biochimica et Biophysica Acta, 398 (1975) 101-110

(C) Elsevier Scientific Publishing Company, Amsterdam - Printed in The Netherlands

BBA 56625

\title{
COMPOSITION AND METABOLISM OF PHOSPHOLIPIDS OF FASCIOLA HEPATICA, THE COMMON LIVER FLUKE
}

\author{
V. OLDENBORG, F. VAN VUGT and L.M.G. VAN GOLDE \\ Laboratory of Veterinary Biochemistry, State University of Utrecht, Biltstraat 172, \\ Utrecht (The Netherlands)
}

(Received December 30th, 1974)

\section{Summary}

1. The phospholipid composition of Fasciola hepatica, the common liver fluke, was compared to that of the liver of the host animals (rats and cattle). Considerable differences were found: monoacyl-sn-glycero-3-phosphorylcholine, hardly detectable in the liver, was found in significant amounts in the parasite. On the other hand, sphingomyelin, a normal constituent in the liver, appears to be absent in the liver fluke. Fasciola hepatica isolated from rat and cow liver had a strikingly similar phospholipid composition.

2. Qualitative and quantitative differences were also found between the fatty acyl constituents of the phospholipids of the parasite and the liver. The major difference was the presence of eicosaenoic and eicosadienoic acids in the parasite, whereas these acids were not detected in the liver.

3. In vitro incubations of Fasciola hepatica in the presence of $\left[{ }^{32} \mathrm{P}\right]$ phosphate and $\left[2{ }^{3} \mathrm{H}\right]$ glycerol resulted in the labelling of all phospholipids of the parasite, except that the ${ }^{3} \mathrm{H}$ label did not incorporate into ethanolamine plasmalogen. This is in agreement with the concept that in animals, glycerol is introduced into plasmalogens via dihydroxyacetonephosphate.

4. Homogenates of liver flukes were found to catalyze the synthesis of phosphatidylcholine from 1,2-diacyl-sn-glycerols and CDPcholine.

5. These results strongly suggest that Fasciola hepatica is capable of synthesizing at least part of its fatty acids and phospholipids.

\section{Introduction}

Fasciola hepatica, the common liver fluke, is a parasite belonging to the class of trematodes. In the adult phase of its life cycle, $F$. hepatica inhabits the liver of mammals, particularly cattle and sheep. It causes considerable damage to the liver parenchyma and bile ducts and, therefore, represents a major problem in veterinary medicine. During the course of an investigation on the inter- 
mediary metabolism of $F$. hepatica, we became interested in the capability of this parasite to synthesize its phospholipids. At present, our knowledge of the composition and origin of the phospholipids of parasites in general and those of $F$. hepatica in particular, is very scarce $[1,2]$.

In the present paper we report the phospholipid composition of $F$. hepati$c a$ in comparison to that of the infected liver tissues. In addition, the fatty acid patterns of the various phospholipids isolated from $F$. hepatica and from the liver of the host animals were compared. In conjunction with this analytical data, the results obtained with in vitro studies on the incorporation of ${ }^{32} \mathrm{P}_{\mathrm{i}}$ and of $\left[2{ }^{3} \mathrm{H}\right]$ glycerol into the phospholipids of $F$. hepatica support the conclusion that this parasite has the capacity to synthesize its phospholipids and/or to induce alterations in lipids obtained from the liver.

Materials and Methods

Growth and isolation of $F$. hepatica

Adult liver flukes from rats were obtained by the following procedures [3] : Eggs from $F$. hepatica were collected at the local slaughterhouse from the bile of naturally infected sheep. The miracidiae from the eggs were used to infect laboratory-reared Limnea trunculata in order to obtain metacercariae. Young male Wistar rats, weighing $40-50 \mathrm{~g}$, were subsequently inoculated orally with 30-35 metacercariae. From 10 weeks post-infection, the rats were killed by stunning and bleeding and the liver flukes were immediately collected from the bile ducts and immersed in standard medium kept at $38^{\circ} \mathrm{C}$. This medium was refreshed three times at intervals of $1 \mathrm{~h}$. Care was taken not to expose the flukes to temperatures below or above $38^{\circ} \mathrm{C}$. Subsequently, the flukes were incubated in fresh standard medium (usually 20 flukes per 1) at $38^{\circ} \mathrm{C}$ to free them from bile components. After $16 \mathrm{~h}$ of incubation the flukes had excreted their intestinal load into the medium and could be used for experiments. The standard medium which was used in all experiments was modified after Van Noordwijk and De Wolf [4] and contained the following components (mM): $\mathrm{NaCl}, 120 ; \mathrm{KCl}, 4 ; \mathrm{CaCl}_{2}, 0.9 ; \mathrm{MgSO}_{4}, 2.4 ; \mathrm{NaHCO}_{3}, 18 ;$ glucose, $5.5 ;$ sodium phosphate buffer ( $\mathrm{pH} 7.5$ ), 4.0. To 11 of medium $10^{6}$ units of benzylpenicilline and $100 \mathrm{mg}$ streptomycine sulphate were added to prevent bacterial growth in the medium.

Liver flukes from cattle were collected at the local slaughterhouse. As soon as possible after the death of an animal the bile ducts were examined for the presence of liver flukes. The flukes were immediately transferred into the standard medium which was kept at $38^{\circ} \mathrm{C}$. Subsequently, the flukes were prepared for the experiments exactly as described for the flukes obtained from rat liver.

\section{Analysis of phospholipids and their plasmalogen analogues}

After removal of the bile components by incubation of the liver flukes overnight, the lipids were extracted via the following procedure: 10 flukes were homogenized in $5 \mathrm{ml}$ of methanol with the aid of an Utraturrax (Janke and Kunkel KG, Germany). Subsequently, $10 \mathrm{ml}$ of chloroform were added and the mixture stirred for $15 \mathrm{~min}$ at room temperature. After centrifugation for 10 
min at room temperature in a clinical centrifuge at maximal speed, the chloroform/methanol supernatant was carefully aspirated and the pellet reextracted twice with $15 \mathrm{ml}$ of chloroform/methanol $(2: 1, \mathrm{v} / \mathrm{v})$. The chloroform $/$ methanol extracts were combined and evaporated in vacuo until dryness. The lipid residue was taken up in $5 \mathrm{ml}$ of chloroform. After two washings with $5 \mathrm{ml}$ of $2 \mathrm{M} \mathrm{KCl}$ and one washing with $5 \mathrm{ml}$ of water, the lipids were subjected to two-dimensional thin-layer chromatography on silica plates impregnated with $2 \%$ magnesium silicate. The thin-layer plates were prepared and activated exactly as described by Broekhuyse [5]. Chloroform/methanol/conc. $\mathrm{NH}_{3} /$ water (90 : $54: 5.5: 5.5$, by vol.) was used as solvent for development in the first direction. After removal of the solvent under a stream of $\mathrm{N}_{2}$, the plate was developed in chloroform/methanol/acetic acid/water $(90: 40: 12: 2, \mathrm{v} / \mathrm{v})$ in the second direction. Detection of the phospholipids was accomplished by exposure to iodine vapour or by spraying with a $0.05 \%$ aqueous solution of Rhodamine 6G. Subsequently, the various phospholipids were scraped from the plate for phosphorus determination according to the procedure of Bartlett [6]. The plasmalogen contents of phosphatidylcholine and phosphatidylethanolamine were determined by subjecting the lipids to methanol/conc. $\mathrm{HCl}(88: 12$, $\mathrm{v} / \mathrm{v})$ prior to development of the plate into the second direction. Phosphorus determination was used to assay the amount of monoacyl-phosphoglycerides derived from the plasmalogens [7]. The various phospholipids were identified by cochromatography with defined standard phospholipids and after mild alkaline hydrolysis by the method of Dawson [8] as modified by Chang and Kennedy [9]. The deacylated phospholipids were separated on Whatman-1 paper chromatograms which were run in ammonium acetate $(1 \mathrm{M}, \mathrm{pH} 7.4) /$ alcohol $(1: 2, \mathrm{v} / \mathrm{v})$. sn-Glycero-3-phosphocholine, sn-glycero-3-phosphoethanolamine, sn-glycero-3-phosphoserine and sn-glycero-3-phosphoinositol were used as reference compounds. Detection was carried out by staining with ammonium molybdate [10], ninhydrin and by radioactivity measurements.

Gas-liquid chromatography of the fatty acyl constituents of the phospholipids of rat liver flukes and rat liver

After two-dimensional thin-layer chromatography the phosphatidylcholine and -ethanolamine fractions were scraped from the plate and transferred into tubes containing $4.5 \mathrm{ml}$ of methanol/conc. $\mathrm{H}_{2} \mathrm{SO}_{4}(95: 5$, w/w $)$. After flushing with $\mathrm{N}_{2}$, the tubes were closed and heated for $2 \mathrm{~h}$ at $70^{\circ} \mathrm{C}$. After cooling, $5.5 \mathrm{ml}$ of water were added and the methylesters were then extracted with three 12.5-ml portions of hexane. The combined hexane layers were washed once with $12.5 \mathrm{ml}$ of water and dried over anhydrous $\mathrm{Na}_{2} \mathrm{SO}_{4}$. Gasliquid chromatography was carried out with a Beckers Research Chromatograph, model 3810 (Beckers, Delft, The Netherlands), equipped with flameionization detection and a $10 \%$ EGSS-X column (Applied Science, U.S.A.) which was operated at $160^{\circ} \mathrm{C}$. The identification was based on comparison with known standard methylesters, supplied by Applied Science and by a semilogarithmic plot of the retention time versus carbon number of the fatty acids [11]. In addition, hydrogenation of the methylesters in hexane using Adams catalyst (Fluka, Switzerland) was used to further identify the unsaturated methylesters. 
In vitro uptake of ${ }^{32} P_{1}$ and $\left[2-^{3} H\right]$ glycerol by $F$. hepatica

After removal of the bile components by incubation of the liver flukes in standard medium as described above, 20 flukes were transferred to $100 \mathrm{ml}$ of fresh medium to which $1 \mathrm{mCi}$ of ${ }^{32} \mathrm{P}_{\mathbf{i}}$ had been added. At the indicated times four flukes were removed from the incubation medium. The lipids were extracted immediately and applied on two-dimensional thin-layer plates, as described above. The detection of the various phospholipids was accomplished by staining with iodine followed by autoradiography. The thin-layer plates were covered with Agfa Osray T4 X-ray plates for the appropriate period of time. The plates were then developed with reagents supplied by Agfa. The radioactive phospholipids were scraped from the plate and transferred into scintillation vials containing $10 \mathrm{ml}$ of Instagel (Packard, U.S.A.). The radioactivity was assayed in -a Packard Tricarb model $2425 \mathrm{~B}$. In some experiments, the flukes were incubated in the presence of $\left[2{ }^{3} \mathrm{H}\right]$ glycerol instead of ${ }^{32} \mathrm{P}_{\mathrm{i}}$. In this case the radioactive phospholipids were detected by a radiochromatogram scanner (Panax Equipment, Great Britain) followed by quantitative assay of the radioactivity by means of liquid scintillation counting.

Synthesis of phosphatidylcholine by homogenates of liver flukes

(a) From 1,2-diacyl-sn-glycerols and CDPcholine. Six flukes were homo-

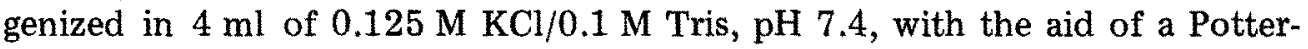
Elvehjem tube and a teflon pestle. The protein content of the homogenate was estimated by the method of Lowry et al. [12]. The formation of phosphatidylcholine from 1,2-diacyl-sn-glycerols and CDP- $\left[\mathrm{Me}^{-{ }^{4}} \mathrm{C}\right]$ choline was assayed in a medium of the following composition: 1,2-diacyl-sn-glycerols ( $4 \mathrm{mM}$, pipetted

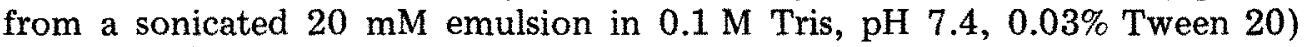
CDP- $\left[\mathrm{Me}^{14} \mathrm{C}\right]$ choline $(4 \mathrm{mM}$, spec. act. $0.25 \mathrm{Ci} / \mathrm{mol}), \mathrm{MgCl}_{2}(10 \mathrm{mM})$, glutathione $(4 \mathrm{mM})$, Tris ' $\mathrm{HCl}(100 \mathrm{mM}, \mathrm{pH} 7.4)$ and the appropriate amounts of liver fluke homogenate. The formation of radioactive phosphatidylcholine was measured after $0,3,6,12$ and $20 \mathrm{~min}$ of incubation by the filter-disc method introduced by Goldfine [13].

(b) By methylation of phosphatidyl-N, N-dimethylethanolamine. The formation of phosphatidylcholine by methylation of phosphatidyl- $N, N$-dimethylethanolamine was studied as follows: $10 \mu \mathrm{mol}$ of phosphatidyl- $N, N$-dimethylethanolamine were suspended by sonication using a MSE-sonifier in $5 \mathrm{ml}$ of a $0.2 \mathrm{M}$ solution of Tris ( $\mathrm{pH} 8.2$ ) containing $20 \mathrm{mg}$ of sodium deoxycholate. 50 $\mu l$ of this suspension, containing $0.1 \mu \mathrm{mol}$ of the substrate, were added to an incubation mixture containing: $1 \mathrm{mM} S$-adenosyl-L $-\left[\mathrm{Me}^{1{ }^{4}} \mathrm{C}\right]$ methionine (spec. act. $2.2 \cdot 10^{5} \mathrm{dpm} / \mu \mathrm{mol}$ ), $50 \mathrm{mM}$ Tris ( $\mathrm{pH} 8.4$ ) and the required amount of liver fluke homogenate. The formation of radioactive phosphatidylcholine was determined at 0,10 and 20 min using the filter-disc method [13] .

\section{Radiochemicals}

Sodium $\left[{ }^{32} \mathrm{P}\right]$ phosphate (spec, act. $165 \mathrm{Ci} / \mathrm{mol}$ ) was purchased from Philips-Duphar, Petten, The Netherlands. $\left[2-^{3} \mathrm{H}\right]$ Glycerol (spec. act. 496 $\mathrm{Ci} / \mathrm{mol}$ ) and $S$-adenosyl-L-[Me $\left.{ }^{14} \mathrm{C}\right]$ methionine (spec. act. $53.1 \mathrm{Ci} / \mathrm{mol}$ ) were obtained from The Radiochemical Centre, Amersham, Great Britain. CDP. $\left[\mathrm{Me}^{1{ }^{14} \mathrm{C}}\right.$ ] choline (spec. act. $40 \mathrm{Ci} / \mathrm{mol}$ ) was bought from N.E.N.-chemicals, Dreieichenhain, Germany. 


\section{Results and Discussion}

Phospholipid composition of $F$. hepatica compared to that of the host liver

Table I shows a comparison of the phospholipid composition of $F$. hepatica with that of the livers of rats and cattle infected with this parasite. The phospholipid pattern of the infected liver tissues is very similar to that reported by Rouser et al. [14] for the normal liver. Evidently, the liver flukes do not cause significant alterations in the phospholipid composition of the liver during their passage through this tissue of the host. Only the percentage of ethanolamine plasmalogen in the infected tissue $(6.8 \%)$ is higher than that reported for the normal liver [15]. Striking differences can be noted between the lipid patterns of the liver flukes and of the liver of the host: (1) Whereas monoacyl-sn-glycero-3-phosphocholine is hardly detectable in the liver, it occurs in significant quantities in the liver flukes. (2) Sphingomyelin, a significant constituent in the liver, is completely absent in the parasite. (3) Phosphatidylethanolamine is more abundant in the liver whereas phosphatidylserine is more enriched in the parasite. This data strongly indicates that the liver fluke does not simply copy the lipid pattern of the liver. Apparently, this parasite has the capacity either to take up lipids from the liver in a very selective way and/or to synthesize its own phopholipids. In this respect, it is very interesting to notice the striking similarity of the lipid compositions of the liver flukes isolated from rat and cattle, respectively.

\section{Fatty acid composition of the major phospholipids of F. hepatica}

Table II presents the fatty acid patterns of phosphatidylcholine and phosphatidylethanolamine of the liver fluke isolated from infected rats. For comparison, the fatty acyl constituents of the corresponding phospholipids in the liver of the infected rats have also been included. It is known that dietary changes profoundly change the fatty acid patterns of liver lipids [16]. Therefore, we limited our studies on the fatty acid composition to $F$. hepatica

PHOSPHOLIPID COMPOSITION (\%) OF F. HEPATICA COMPARED TO THAT OF THE LIVER OF THE HOST

\begin{tabular}{|c|c|c|c|c|}
\hline & \multicolumn{2}{|l|}{ F. hepatica* } & \multicolumn{2}{|c|}{ Liver $* *$} \\
\hline & From cattle & From rat & Cattle & Rat \\
\hline Monoacyl-sn-glycero-3-phosphocholine & 5.6 & 4.2 & 0.2 & 0.2 \\
\hline Phosphatidylcholine & 46.8 & 49.2 & $\mathbf{5 2 . 5}$ & 52.0 \\
\hline Choline plasmalogen & 3.4 & 3.2 & $\mathbf{3 . 0}$ & 0.9 \\
\hline Sphingomyelin & - & - & 4.2 & 3.4 \\
\hline Phosphatidylserine & 13.6 & 13.6 & 4.6 & 4.8 \\
\hline Phosphatidylinositol & 6.9 & 6.9 & 9.6 & 8:8 \\
\hline Phosphatidylethanolamine & 18.2 & 16.5 & 19.2 & 20.5 \\
\hline Ethanolamine plasmalogen & 3.9 & 3.6 & 4.8 & 6.8 \\
\hline Cardiolipin & 1.7 & 2.8 & 1.8 & 2.7 \\
\hline
\end{tabular}

* Average value of ten experiments.

** Average value of four experiments. 
TABLE II

FATTY ACID COMPOSITION (\%) OF PHOSPHATIDYLCHOLINE AND PHOSPHATIDYLETHANOLAMINE FROM F. HEPATICA AND FROM RAT LIVER

Averages of three experiments are presented. + , trace amounts present

\begin{tabular}{lcccc}
\hline Fatty acids & \multicolumn{2}{l}{ Phosphatidylcholine } & & \multicolumn{2}{l}{ Phosphatidylethanolamine } \\
\cline { 2 - 3 } & Liver & F.hepatica & Liver & F. hepatica \\
\hline $16: 0$ & 26.8 & 39.3 & 28.2 & 9.9 \\
$18: 0$ & 20.1 & 19.6 & 30.3 & 38.5 \\
$18: 1$ & 8.6 & 9.5 & 9.0 & 7.4 \\
$18: 2$ & 20.4 & 5.8 & 9.6 & 4.7 \\
$20: 1$ & - & 7.1 & - & 4.9 \\
$20: 2$ & - & 4.6 & 10.3 & 0.7 \\
$20: 4$ & 23.9 & 13.6 & 12.8 & 22.0 \\
$22: 6$ & + & + & & 11.7 \\
\hline
\end{tabular}

isolated from rats which had been fed with a regular laboratory chow-pellet diet. The fatty acid compositions of phosphatidylcholine and phosphatidylethanolamine from the liver are in good agreement with data reported for these phospholipids by other investigators [17] : phosphatidylethanolamine is richer than phosphatidylcholine in stearic and docosahexaenoic acid whereas phosphatidylcholine contains higher amounts of linoleic and arachidonic acid. The fatty acids of the corresponding phospholipids in the liver fluke are quite different: in phosphatidylcholine there is an increase of palmitic acid and a decrease of linoleic and arachidonic acid. Also qualitative differences can be noted; eicosaenoic $\left(C_{20: 1}\right)$ and eicosadienoic $\left(C_{20: 2}\right)$ acid occur in significant amounts in the parasite, whereas these acids cannot be detected in the liver. It is of interest that another trematode, Schistosoma mansoni, also contains significant amounts of $\mathrm{C}_{20: 1}$ and $\mathrm{C}_{20: 2}[18,19]$. Presumably, these acids are formed by the parasite by elongation of octadecenoic $\left(\mathrm{C}_{18: 1}\right)$ and octadecadienoic $\left(C_{18: 2}\right)$ acid, respectively. In this light it is relevant to mention that another trematode, $S$. mansoni, and a cestode, Hymenolepis diminuta, have been shown to possess the capacity to elongate fatty acids $[19,20]$. Further experiments are in progress to investigate the elongation system of $F$. hepatica. Also for phosphatidylethanolamine, the major qualitative difference is the occurrence of $\mathrm{C}_{20: 1}$ and $\mathrm{C}_{20: 2}$ in the parasite and the absence of these fatty acids in the liver. Similarly as found for the polar head group of the phospholipids, the fatty acid pattern of the phospholipids of the parasite is not merely a reflection of that of the phospholipids of the liver. Evidently, $F$. hepatica has the capacity to synthesize at least part of its fatty acids de novo or to introduce modifications in fatty acids obtained from the liver.

In vitro uptake of ${ }^{32} P_{1}$ and $\left[2{ }^{3} H\right]$ glycerol by F. hepatica

Incubation of $F$. hepatica isolated from rats in standard medium to which ${ }^{32} \mathrm{P}_{\mathrm{i}}$ had been added resulted in the labelling of all phospholipids of this parasite. In Fig. 1 the rate of incorporation of ${ }^{32} \mathrm{P}_{\mathrm{i}}$ into the various phospholipids is studied. Phosphatidylinositol was found to have the highest specific activity over the whole incubation period. Probably, the specific activity of 


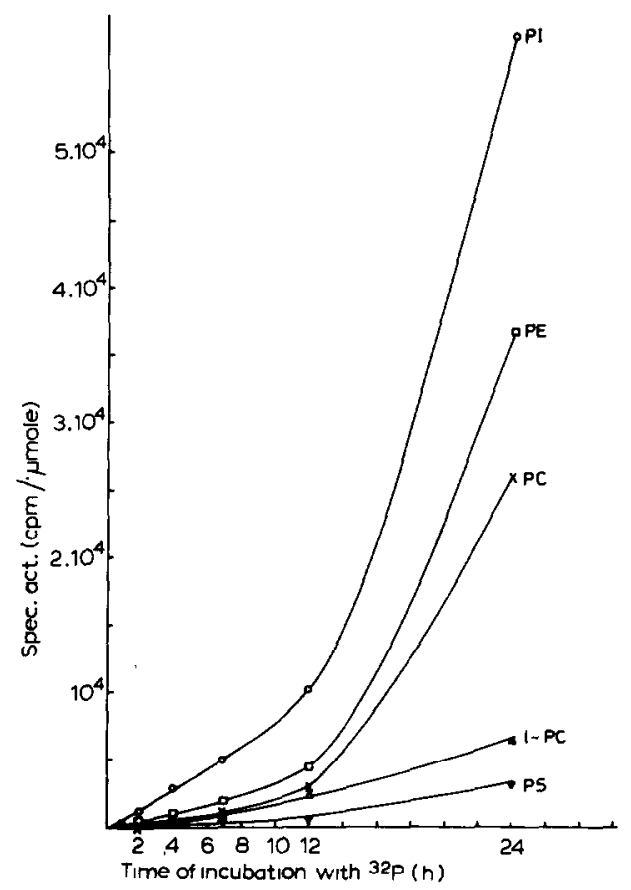

Fig. 1. In vitro uptake of ${ }^{32} \mathrm{P}_{\mathrm{i}}$ by $F$. hepatica isolated from rats. ${ }^{-}-0$, phosphatidylinositol (PI); $\square-\square$, phosphatidylethanolamine (PE); $X-X$, phosphatidylcholine (PC); $\triangle \longrightarrow$, monoacylsn-gly cero-3-phosphocholine (1-PC); $\triangle \longrightarrow \triangle$, phosphatidylserine (PS).

phosphatidic acid is even higher than that of phosphatidylinositol, but its specific activity could not be measured accurately due to the extreme small amounts of this phospholipid in $F$. hepatica. Phosphatidylethanolamine and phosphatidylcholine were labelled at an almost constant rate during the first $7 \mathrm{~h}$ of incubation, after which time a strong increase in the rate of incorporation was noted. Similar observations were noted by Webb and Mettrick [21] who studied the uptake of ${ }^{32} \mathrm{P}_{\mathrm{i}}$ and by Buteau and Fairbairn [22] who followed the incorporation of $\left[{ }^{14} \mathrm{C}\right]$ glycerol into the phospholipids of the rat tape-worm, $H$. diminuta.

Monoacyl-sn-glycero-phosphocholine and phosphatidylserine were labelled at a much slower rate than the other phospholipids of $F$. hepatica, probably because of a much slower turn-over. After $24 \mathrm{~h}$ of incubation the percentage of label incorporated into the choline-containing lipids, phosphatidylserine and cardiolipin was smaller than the weight percentage of these lipids (Table III). On the other hand, the percentage of label incorporated into phosphatidylinositol and phosphatidylethanolamine exceeded the weight percentage of these phospholipids. The higher labelling of phosphatidylinositol and phosphatidylethanolamine may be related to a preliminary observation that these phospholipids are enriched in the eggs produced by the liver fluke. Incubation of liver flukes in the presence of $\left[2-^{3} \mathrm{H}\right]$ glycerol resulted in a similar labelling pattern of the various phospholipids, with the following exceptions: (1) Phosphatidylserine is labelled more strongly by glycerol than by phosphate. (2) Ethanol- 
DISTRIBUTION (\%) OF RADIOACTIVITY AMONG THE PHOSPHOLIPIDS OF F. HEPATICA AFTER 24 h INCUBATION IN THE PRESENCE OF ${ }^{32} P_{i}$ OK $\left[2-{ }^{3} H\right] G L Y C E R O L$

+ , trace amounts present.

\begin{tabular}{lrrr}
\hline & ${ }^{32} \mathrm{P}_{1}$ & {$\left[2-{ }^{3} \mathrm{H}\right]$ Glycerol } & Weight distribution \\
\hline Monoacyl-sn-glycero-3-phosphocholine & 2.6 & 1.0 & 4.2 \\
Phosphatidylcholine & 37.2 & 37.0 & 49.2 \\
Phosphatidylserine & 1.9 & 7.8 & 13.6 \\
Phosphatidylinositol & 22.4 & 23.9 & 6.9 \\
Phosphatidylethanolamine & 29.0 & 29.4 & 16.5 \\
Ethanolamine plasmalogen & 7.1 & 0.8 & 3.6 \\
Cardiolipin & + & + & 2.8 \\
\hline
\end{tabular}

amine plasmalogen is labelled by ${ }^{32} \mathrm{P}_{\mathrm{i}}$ but hardly by $\left[2 \cdot{ }^{3} \mathrm{H}\right]$ glycerol. These observations are in excellent agreement with the concept $[23,24]$ that in animals glycerol is introduced into alkyl lipids via dihydroxyacetonephosphate. Since it is known that ethanolamine plasmalogen is derived from 1-alkyl-2acyl-sn-glycero-3-phosphoethanolamine $[25],\left[2-{ }^{3} \mathrm{H}\right]$ glycerol should indeed loose its ${ }^{3} \mathrm{H}$ label upon incorporation into ethanolamine plasmalogen. It cannot be excluded, however, that the biosynthesis of plasmalogens in $F$. hepatica proceeds via another mechanism as has been described for mammalian tissues [25], since the metabolism of the fluke is essentially anaerobic and because there is evidence [20] that helminths might lack the capacity to desaturate fatty acids. These results obtained in experiments on the uptake of ${ }^{32} P_{i}$ and $\left[2{ }^{3} \mathrm{H}\right]$ glycerol into the phospholipids of $F$. hepatica during in vitro incubations clearly demonstrate that this parasite is equipped with the machinery to synthesize and renew its own phospholipids de novo.

\section{Synthesis of phosphatidylcholine by homogenates of $F$. hepatica}

The synthesis of phosphatidylcholine in mammalian cells proceeds via the CDPcholine pathway, established by Kennedy [26]. An additional pathway for

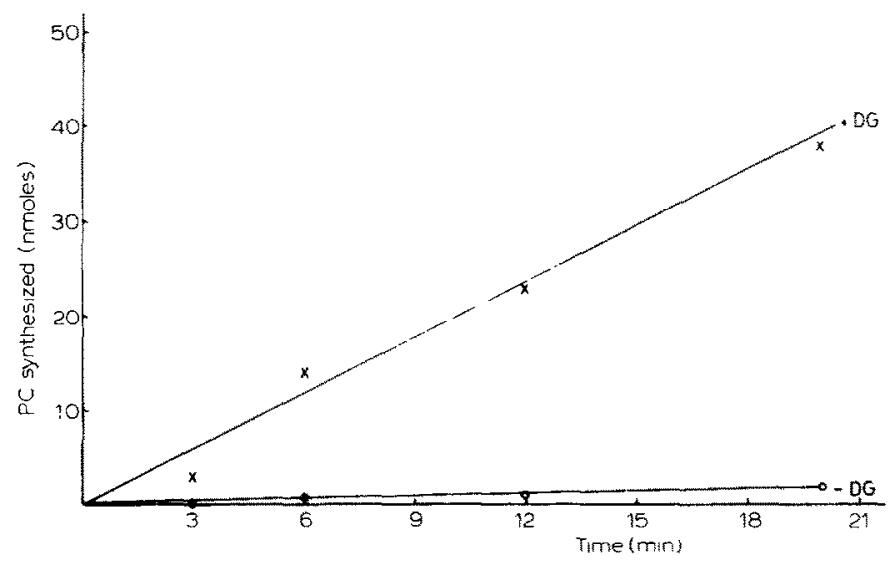

Fig. 2. Synthesis of phosphatidylcholine from 1,2-diacyl-sn-glycerols and CDP-[Me-14 $\mathrm{C}]$ choline by homogenates of $F$. hepatica. Detalis of the incubation mixture have been mentioned in Materials and Methods. DG, 1,2 diacyl-sn-gly cerols. 
the synthesis of phosphatidylcholine, viz. the N-methylation of phosphatidylethanolamine [27-29], has been shown to be almost exclusively confined to the liver [30], where it is believed to be operative mainly in the production of hexaenoic lecithins $[31,32]$. It was thought of interest to investigate whether the liver fluke synthesizes phosphatidylcholine via CDPcholine or via N-methylation. Fig. 2 shows that homogenates of $F$. hepatica contain choline phosphotransferase (EC 2.7.8.2): the synthesis of phosphatidylcholine from added 1,2diacyl-sn-glycerols and CDP-[Me- $\left.{ }^{14} \mathrm{C}\right]$ choline was found to be proportional to time and amount of protein. However, in strong contrast to rat liver microsomes, homogenates of $F$. hepatica were not able to convert dimethyl phosphatidylethanolamine into phosphatidylcholine by N-methylation. Apparently, the liver fluke only uses the de novo synthesis via CDPcholine for the formation of phosphatidylcholine. In future studies it will be of interest to investigate the presence of a deacylation-reacylation cycle [33] in $F$. hepatica, especially in view of the exceptionally high level of monoacyl-sn-glycero-3-phosphocholine in this parasite.

\section{Acknowledgements}

The present investigations have been carried out under the auspices of the Netherlands Organization for Chemical Research (S.O.N.) and with the financial aid from the Netherlands Organization for the Advancement of Pure Research (ZWO). The authors wish to thank Drs C.A. Ladage from A.C.F. Chemiefarma N.V. (Maarssen, The Netherlands) for the supply of $F$. hepatica metacercariae. The authors gratefully acknowledge the help of Drs J.T.G. van Loor, Drs S. Klapwijk and their coworkers of the Municipal Slaughterhouse of Dordrecht (The Netherlands) in collecting $F$. hepatica from cattle.

\section{References}

1 Von Brand, Th. (1972) Parasiten Physiologie, pp. 70-72 Gustav Fischer Verlag. Stuttgart

2 Von Brand, Th. (1972) Parasiten Physiologie, pp. 107-112, Gustav Fischer Verlag, Stuttgart

3 Boray, J.C. (1963) Proceedings of the Symposium on the evaluation of anthelmintics in Hannover (Soulsby, E.J.L., ed.), pp. 34-45, Merck, Sharp and Dohme International, New York

4 Van Noordwijk, J. and de Wulf, J.N. (1963) Acta Physiol. Pharmacol. Neerl. 12, 30-47

5 Broekhuyse, R.M. (1968) Biochim. Biophys. Acta 152, 307-315

6 Bartlett, G.R. (1959) J. Biol. Chem. 234, 466-468

7 Van Golde, L.M.G., Prins, R.A., Franklin-Klein, W. and Akkermans-Kruyswijk, J. (1973) Biochim. Biophys. Acta 326, 314-323

8 Dawson, R.M.C. (1954) Biochim. Biophys. Acta 14, 374-379

9 Chang, Y. and Kennedy, E.P. (1967) J. Biol. Chem. 242, 516-519

10 Borrows, S., Grylls, F.S.M. and Harrison, J.S. (1952) Nature 170, 800-801

11 James, A.T. and Martin, A.J.P. (1952) Biochem. J. 50, 679-690

12 Lowry, O.H., Rosebrough, N.J., Farr, A.L. and Randall, R.J. (1951) J. Biol. Chem. 193, 265-275

13 Goldfine, H. (1966) J. Lipid. Res. 7. 146-149

14 Rouser, G., Simon, G. and Kritchevsky, G. (1969) Lipids 4, 599-606

15 Horrocks, L.A. (1972) Ether Lipids, Chemistry and Biology (Snyder, F., ed.), pp. 177-272, Academic Press, New York

16 Van Golde, L.M.G. and Van Deenen, L.L.M. (1966) Biochim. Biophys. Acta 125, 496-509

17 Getz, G.S., Bartley, W., Stirpe, F., Notton, B.M. and Renshaw, A. (1961) Biochem. J. 80, 176-181

18 Smith, T.M., Brooks, T.J. and White, H.B. (1969) Lipids $4,31-36$

19 Meyer, F., Meyer, H. and Bueding, E. (1970) Biochim. Biophys. Acta 210, 257-266

20 Jacobsen, N.S. and Fairbairn, D. (1967) J. Parasitol. 53, 355-361 
21 Webb, R.A. and Mettrick, D.F. (1971) Can. J. Biochem. 49, 1209-1212

22 Buteau, G.H. and Fairbairn, D. (1969) Exp. Parasitol. 25, 265-275

23 Snyder, F., Malone, B. and Blank, M.L. (1970) J. Biol. Chem. 245, 1790-1799

24 Hajra, A.K. (1970) Biochem. Biophys. Res. Commun. 39, 1037-1044

25 Wykle, R.L., Blank, M.L., Malone, B. and Snyder, F. (1972) J. Biol. Chem. 247, 5442-5447

26 Kennedy, E.P. (1961) Fed. Proc. 20, 934-940

27 Bremer, J. and Greenberg, D.M. (1961) Biochim. Biophys. Acta 46, 205-216

28 Artom, C. and Lofland, H.B. (1960) Biochem. Biophys. Res. Commun. 3, 244--247

29 Gibson, K.D., Wilson, J.D. and Udenfriend, S. (1961) J. Biol. Chem. 236, 673-679

30 Bjфrnstad, P. and Bremer, J. (1966) J. Lipid Res. 7, 38-45

31 Arvidson, G. (1968) Eur. J. Biochem. 4, 478-486

32 Lyman, R.L., Hopkins, S.M., Sheehan, G. and Tinoco, J. (1968) Biophys. Acta 176, 86--94

33 Lands, W.E.M. (1960) J. Biol. Chem, 2233-2237 